BULLETIN OF THE

AMERICAN MATHEMATICAL SOCIETY

Volume 80, Number 6, November 1974

\title{
MANIFOLDS WITH FUNDAMENTAL GROUP A GENERALIZED FREE PRODUCT. I
}

\author{
BY SYLVAIN E. CAPPELL1
}

Communicated by William Browder, February 26, 1974

This announcement describes new methods in the study and classification of differentiable, P1 or topological manifolds with infinite fundamental group. If, for example, $Y^{n+1}$ is a closed manifold with $\pi_{1} Y=G_{1} *_{H} G_{2}$, $n \geqslant 4$, there is a decomposition $Y=Y_{1} \cup_{X} Y_{2}$ with $\pi_{1}\left(Y_{1}\right)=G_{1}, \pi_{1}\left(Y_{1}\right)=$ $G_{2}, \pi_{1}(X)=H$. For large classes of fundamental groups, the codimension one splitting theorems of $[\mathbf{C} 3]$, extending results of $[\mathrm{B} 1],[\mathrm{B} 2],[\mathrm{BL}],[\mathbf{F H}],[\mathbf{L}]$, [W2], reduced the classification of manifolds homotopy equivalent to $Y$ to the classifications of the manifolds homotopy equivalent to $Y_{1}$ and to $Y_{2}$. However, the construction in [C4] and [C5] of manifolds $V$ simple and tangentially homotopy equivalent to $R P^{4 k+1} \# R P^{4 k+1}, k>0$, but $V$ is not a nontrivial connected sum, demonstrated in a strong way the existence of unsplittable manifolds and homotopy equivalences. 2

In the present announcement we get around these difficulties by, roughly speaking, adapting methods of [C3] to construct an abelian group we call the unitary nilpotent group which depends on $H, G_{1}, G_{2}$ and which acts freely on the set of manifolds equipped with homotopy equivalences to $Y$, with each coset of this action containing a unique split manifold. Thus, the classification of manifolds homotopy equivalent to $Y$ is reduced to computing UNil groups and to classifying split homotopy equivalences. In this setting, all earlier splitting theorems are reinterpreted as showing that for certain $H, G_{1}, G_{2}$, the unitary nilpotent group vanishes. However, for $H=0, Z_{2} \subset G_{1}, G_{2} \neq 0$, $n=4 k$ and $Y$ orientable, the corresponding unitary nilpotent group is not finitely generated [C4], [C6].

The unitary nilpotent groups are 2-primary and are defined algebraically in [C6] and depend only on the ring with involution $Z[H]$, the $Z[H]$-bi-

AMS (MOS) subject classifications (1970). Primary 57A35, 57B10, 57C35, 57D40, 57D65, 57D80; Secondary 57D20, $18 \mathrm{~F} 25$.

1 The author is an A. P. Sloan fellow and was partially supported by an NSF grant.

2 Note also our construction in [C4] and [C5] of counterexamples to a splitting theorem of Miščenko [M]. 
modules with involution $Z\left[\hat{G}_{i}\right]=Z\left[G_{i}\right] / Z[H], i=1,2$, and $n$ modulo 4 . They satisfy a semiperiodicity of period 2 described in [C6].

The splitting theorems of [C3] led to the computation, in many cases, of the Wall surgery obstruction groups of generalized free products [C2]. Earlier the splitting theorems of [FH] were used in [S1], [W2] to compute the surgery groups of $Z \times H$. Using our new unitary nilpotent groups we extend our results in [C6] to obtain general Mayer-Vietoris type sequences for Wall groups of generalized free products.

Splitting problems for codimension one submanifolds $X$ of $Y$ with $Y-X$ having one component will be treated using similar methods in [C7] and will be used to obtain further results on Wall groups in [C6]. The present results imply, in many cases, Novikov's conjecture on the homotopy invariance of higher signatures [C8]. Further applications to decompositions of manifolds and Poincaré duality spaces, codimension one submanifolds, diffeomorphism groups etc. will be presented elsewhere.

There are relative forms of all the results described below, but for simplicity we deal here only with the absolute case. For the remainder of this paper we fix the following notation. Let $Y$ be a closed manifold or Poincare complex of dimension $n+1, Y=Y_{1} \cup_{X} Y_{2}, X=\partial Y_{1}=\partial Y_{2} \neq \varnothing, Y_{1}, Y_{2}$, $X$ connected manifolds or Poincaré complexes with $\pi_{1}(X) \rightarrow \pi_{1}\left(Y_{i}\right)$ injective, $i=1,2$, or equivalently $\pi_{1}(X) \rightarrow \pi_{1}(Y)$ injective. Set $H_{1}=\pi_{1}(X)$, $G_{i}=\pi_{i}\left(Y_{i}\right), i=1,2$. Let

$$
\begin{aligned}
\varphi & =\left(Z[H] ; Z\left[\hat{G}_{1}\right], Z\left[\hat{G}_{2}\right]\right) \\
\beta & =\operatorname{Ker}\left(\widetilde{K}_{0}(Z[H]) \rightarrow \widetilde{K}_{0}\left(Z\left[G_{1}\right]\right) \oplus \widetilde{K}_{0}\left(Z\left[G_{2}\right]\right)\right),
\end{aligned}
$$

and

$$
\mu=\operatorname{Image}\left(\mathrm{Wh}\left(G_{1}\right) \oplus \mathrm{Wh}\left(G_{2}\right) \rightarrow \mathrm{Wh}\left(G_{1} *_{H} G_{2}\right)\right)
$$

As usual the group-rings $Z[H], Z\left[G_{1}\right], Z\left[G_{2}\right]$ have involutions determined by the first Stiefel-Whitney class of $Y$. Let $Z\left[\hat{G}_{i}\right]$ denote the quotient $Z[H]$ bimodule with involution $Z\left[G_{i}\right] / Z[H]$; note that additively $Z\left[\hat{G}_{i}\right]$ is generated by elements of $G_{i}$ not in $H, i=1,2$. The 2-primary abelian groups $\mathrm{UNil}_{n}^{h}(\varphi)$ are defined in [C6] ; for $n$ even, or if $Z[H]$ is a regular ring or just coherent of finite global homological dimension, we also define there abelian 2-primary groups $\mathrm{UNil}_{n}^{s}(\varphi)$.

The homotopy equivalence $f: W \rightarrow Y, W$ a closed differentiable (resp. 
P1, topological) manifold, is called splittable along $X$ if $f$ is homotopic to a map, which we continue to denote by $f$, which is transverse regular to $X$, so that $M=f^{-1}(X)$ is a differentiable (resp. P1, topological) submanifold of $W$, with $f$ restricting to a homotopy equivalence $M \rightarrow X$. This would imply, as $\pi_{1} X \rightarrow \pi_{1} Y$ is assumed injective, that $f^{-1}\left(Y_{i}\right) \rightarrow Y_{i}, i=1,2$, are also homotopy equivalences.

Theorem 1 describes the obstruction to splitting a manifold up to $h$-cobordism, and Theorem 2 gives a realization result for this obstruction.

THEOREM 1 ( $h$-SPLITTING THEOREM). Let $f: W \rightarrow Y$ be a homotopy equivalence with $W$ a closed differentiable, P1 (resp. or topological) manifold of dimension $n+1, n \geqslant 4$, and $X^{n} \subset Y$ with, as above, $\pi_{1}\left(X_{-}\right)=H$, $\pi_{1}(Y)=G_{1} *_{H} G_{2}$. If $n=4$, assume further that $X$ is a P1 (resp. topological) manifold with $H=0$ or $H$ finite of odd order. Then there is an h-cobordism $\left(V ; W, W^{\prime}\right)$ with the induced homotopy equivalence $f^{\prime}: W^{\prime} \rightarrow Y$ splittable along $X$ if and only if $\chi^{h}(f)$ and $\bar{\Phi}(\tau(f))$ are zero, where $\chi^{h}(f) \in \mathrm{UNil}_{n+2}^{h}(\varphi)$ and $\bar{\Phi}(\tau(f)) \in H^{n+1}\left(Z_{2} ; \beta\right)$ are invariants of the homotopy class of $f$.

In Theorem $1, \bar{\Phi}(\tau(f))$ is the cohomology class represented by $\Phi(\tau(f))$ for $\tau(f)$ the Whitehead torsion of $f$ and $\Phi: \operatorname{Wh}\left(G_{1} *_{H} G_{2}\right) \rightarrow \beta$ the map defined in [W1]. Given $f: W \rightarrow Y$ as in Theorem 1 , if $\left(V ; W, W^{\prime}\right)$ is an $h$-cobordism, then letting $f^{\prime}: W^{\prime} \rightarrow Y$ denote the induced homotopy equivalence, $\chi\left(f^{\prime}\right)=\chi(f)$ and $\bar{\Phi}(\tau(f))=\bar{\Phi}\left(\tau\left(f^{\prime}\right)\right)$. Even for $n \leqslant 4$, the vanishing of $\chi^{h}(f)$ and $\Phi(\tau(f))$ are necessary conditions for $W$ to be $h$-cobordant to a split manifold.

Let $S_{F}^{h}(Y)$ denote equivalence classes of pairs $(W, f)$ where for $F=O$ (resp. $F=\mathrm{P} 1$, or $F=$ Top), $W$ a closed differentiable (resp. $\mathrm{P} 1$, or topological) manifold, and $f: W \rightarrow Y$ a homotopy equivalence [S2]. Here $(W, f)$ is equivalent to $\left(W^{\prime}, f^{\prime}\right)$ if there is an $h$-cobordism $\left(V ; W, W^{\prime}\right)$ with $f$ and $f^{\prime}$ extending to a homotopy equivalence $V \rightarrow Y$. The maps $\chi$ and $\bar{\Phi}$ of Theorem 1 induce maps

$$
\chi: S_{F}^{h}(Y) \rightarrow \mathrm{UNil}_{n+2}^{h}(\varphi) \text { and } \bar{\Phi}: S^{h}(Y) \rightarrow H^{n+1}\left(Z_{2} ; \beta\right) .
$$

THEOREM 2 ( $h$-SPLITTING OBSTRUCTION REALIZATION). Let $Y$ be a Poincaré complex or manifold of dimension $n+1, n \geqslant 4, Y=Y_{1} \cup_{X} Y_{2}$, $\pi_{1} Y=G_{1} *_{H} G_{2}, \pi_{1}(X)=H$ as above. Then there is a free action of $\mathrm{UNil}_{n+2}^{h}(\varphi)$ on $S_{F}^{h}(Y), F=O, \mathrm{P} 1$, or Top, where for $x \in S_{F}^{h}(Y)$ and 
$\alpha \in \mathrm{UNil}_{n+2}^{h}(\varphi), \chi^{h}(\alpha x)=\chi^{h}(x)+\alpha$ and $\Phi(\alpha x)=\Phi(x)$. Also, $\alpha x$ and $x$ are normally cobordant.

Let $\operatorname{Split}_{F}^{h}(Y ; X)$ denote the set of pairs $(W, f)$, where for $F=O$ (resp. $F=\mathrm{P} 1$ or Top), $W$ is a closed differentiable (resp. P1 or topological) manifold, and $f: W \rightarrow Y$ is a homotopy equivalence split along $X \subset Y$, classified up to split $h$-cobordisms. There is a canonical "forgetful" map

$$
\xi: \operatorname{Split}_{F}^{h}(Y ; X) \rightarrow S_{F}^{h}(Y) \text {. }
$$

If $Y$ is a manifold with $Y=Y_{1} \cup_{X} Y_{2}=Y_{1}^{\prime} \cup_{X^{\prime}} Y_{2}^{\prime}, \pi_{1}\left(Y_{i}\right)=\pi_{1}\left(Y_{i}^{\prime}\right)$ $=G_{i}, i=1,2, X$ and $X^{\prime}$ codimension one submanifolds with $\pi_{1}(X)=$ $\pi_{2}\left(X^{\prime}\right)=H$, then $\xi\left(\operatorname{Split}_{F}^{h}(Y ; X)\right)=\xi\left(\operatorname{Split}_{F}^{h}\left(Y, X^{\prime}\right)\right)$.

Theorem 3. Let $Y$ be as in Theorem 1. If $H^{i}\left(Z_{2} ; \beta\right)=0, i>1$, then there is a one-to-one correspondence for $F=O, \mathrm{P} 1$ or Top,

$$
S_{F}^{h}(Y) \stackrel{p \times \chi^{h}}{\longrightarrow} \operatorname{Split}_{F}^{h}(Y ; X) \times \mathrm{UNil}_{n+2}^{h}(\varphi) .
$$

Moreover, $p \xi$ is the identity map of $\operatorname{Split}_{F}^{h}(Y ; X)$. If for $x, y \in S_{F}^{h}(Y)$, $p(x)=p(y)$, then $x$ and $y$ are normally cobordant.

There is a map of $\mathrm{UNil}_{n}^{h}(\varphi)$ to $H^{n}\left(Z_{2} ; \widetilde{\mathrm{Nil}}(\varphi)\right)$ which is the analogue of a map in the Rothenburg exact sequence [S1]. The group $\widetilde{N} i l(\varphi)$ of reduced nilpotent objects, which is defined and studied in [W1], vanishes if $Z[H]$ is a regular ring or just coherent of finite global homological dimension.

THEOREM 4 (SPlitTING THEOREM, FIRST FORM). Hypothesis as in Theorem 1: Then $f: W \rightarrow Y$ is splittable along $X$ if and only if $\tau(f) \in \mu$, $\chi^{h}(f)=0$ and an obstruction $\kappa(f)$ defined in that case is also zero,

$$
\kappa(f) \in \operatorname{Coker}\left(\mathrm{UNil}_{n+3}^{h}(\varphi) \rightarrow H^{n+3}\left(Z_{2} ; \widetilde{\operatorname{Nil}}(\varphi)\right)\right) .
$$

The last 2 obstructions of Theorem 4 can be combined into one as follows:

THEOREM 5 (SPLITTING THEOREM, SECOND FORM). Hypothesis as in Theorem 1 and assume further that $n$ is even or that $Z[H]$ is a regular ring or just a coherent ring of finite global homological dimension. Then $f: W \rightarrow Y$ is splittable along $X$ if and only if $\tau(f) \in \mu$ and an obstruction $\chi^{s}(f)$, defined in that case, is zero. Here, $\chi^{s}(f) \in \operatorname{UNil}_{n+2}^{s}(\varphi)$ is an invariant of the homotopy class of $f$.

Let $S_{F}^{s}(Y)$ denote the set of pairs $(W, f)$ with $F=O$ (resp. P1 or 
Top), $W$ a closed differentiable (resp. P1 or topological) manifold and $f: W \rightarrow Y$ a simple homotopy equivalence. Here $(W, f)$ is equivalent to $\left(W^{\prime}, f^{\prime}\right)$ if there is a diffeomorphism (resp. P1 homeomorphism or topological homeomorphism) $g: W \rightarrow W^{\prime}$ with $f^{\prime} g$ homotopic to $f$ [S2]. The map $\chi^{s}$ of Theorem 5 induces a map $\chi^{s}: S_{F}^{s}(Y) \rightarrow \mathrm{UNil}_{n+2}^{s}(\varphi)$.

THEOREM 6 (SPlitTing OBSTRUCTION REALizATION). For $Y$ as in Theorem 2 with $n$ even or with $Z[H]$ a regular ring or just a coherent ring of finite global homological dimension, $\chi^{s}: S_{F}^{s}(Y) \rightarrow \mathrm{UNil}_{n+2}^{s}(\varphi)$ maps each normal cobordism class in $S_{F}^{s}(Y)$ surjectively onto $\mathrm{UNil}_{n+2}^{s}(\varphi), F=O, \mathrm{P} 1$ or Top. Further, if $n$ is even, or if $H=0$, or if $\operatorname{Wh}\left(G_{1} *_{H} G_{2}\right)=0$, then there is a free action of $\mathrm{UNil}_{n+2}^{s}(\varphi)$ on $S_{F}^{s}(Y)$ with $\chi^{s}(\alpha x)=\alpha+\chi^{s}(x)$ for $\alpha \in \mathrm{UNil}_{n+2}^{s}(\varphi), x \in S_{F}^{s}(Y)$.

Let $S_{F}^{0}(Y)$ denote the set of pairs $(W, f)$ with $F=O$ (resp. P1 or Top), $W$ a closed differentiable (resp. P1 or topological) manifold, and $f: W \rightarrow Y$ a homotopy equivalence with $\tau(f) \in \mu$. Here $(W, f)$ is equivalent to $\left(W^{\prime}, f^{\prime}\right)$ if there is an $h$-cobordism $\left(V ; W, W^{\prime}\right)$ with Whitehead torsion in $\mu$ and with $f$ and $f^{\prime}$ extending to a homotopy equivalence $V \rightarrow Y$. If

$$
H^{i}\left(Z_{2} ; \operatorname{Wh}\left(G_{1}\right) \oplus_{\mathrm{Wh}(H)} \operatorname{Wh}\left(G_{2}\right)\right)=0, \quad i>1,
$$

then $S_{F}^{0}(Y)=S_{F}^{s}(Y)$.

There is a "forgetful" map $\xi: \operatorname{Split}_{F}^{h}(Y ; X) \rightarrow S_{F}^{0}(Y)$.

THEOREM 7. Hypothesis as in Theorem 1. If $n$ is even, or if $Z[H]$ is a regular ring or just coherent of finite global homological dimension, for $F=0, \mathrm{Pl}$ or Top there is a one-to-one correspondence

$$
S_{F}^{0}(Y) \stackrel{p \times \chi^{s}}{\longrightarrow} \operatorname{Split}_{F}^{h}(Y ; X) \times \mathrm{UNil}_{n+2}^{s}(\varphi) .
$$

Note that many of the above results cover in some cases $n=4$. The present methods together with the special low-dimensional methods of [CS] give general results on stable splitting problems for $n=4$.

\section{BIBLIOGRAPHY}

[B1] W. Browder, Embedding 1-connected manifolds, Bull. Amer. Math. Soc. 72 (1966), 225-231; erratum, ibid. 72 (1966), 736. MR 32 \#6467.

[B2] - Manifolds with $\pi_{1}=Z$, Bull. Amer. Math. Soc. 72 (1966), $238-$ 244. MR $32 \# 8350$.

[BL] W. Browder and J. Levine, Fibering manifolds over a circle, Comment. Math. Helv. 40 (1966), 153-160. MR 33 \#3309. 
[C1] S. E. Cappell, A splitting theorem for manifolds and surgery groups, Bull. Amer. Math. Soc. 77 (1971), 281-286. MR 44 \#2234.

[C2 ] - Mayer-Vietoris sequences in Hermitian $K$-theory, Proc. Battelle $K$ theory Conference, Lecture Notes in Math., vol. 343, Springer-Verlag, Berlin and New York, 1973, pp. 478-512.

[C3] - A splitting theorem for manifolds (to appear).

[C4] - Splitting obstructions for Hermitian forms and manifolds with $Z_{2} \subset \pi_{1}$, Bull. Amer. Math. Soc. 79 (1973), 909-914.

[C5] - Connected sums of manifolds, Topology (to appear).

[C6] Unitary nilpotent groups and Hermitian K-theory. I, Bull. Amer. Math. Soc. 80 (1974),

[C7] - Manifolds with fundamental group a generalized free product. II (to appear).

[C8] - On the homotopy invariance of higher signatures (to appear).

[CS] S. E. Cappel and J. L. Shaneson, Four-dimensional surgery and applications, Comment. Math. Helv. 46 (1971), 500-528. MR 46 \#95.

[FH] F. T. Farrell and W. C. Hsiang, Manifolds with $\pi_{1}=G \times_{\alpha} T$, Amer. J. Math. (to appear).

[L] R. Lee, Splitting a manifold into two parts, Mimeographed notes, Institute for Advanced Study. Princeton, N. J., 1969.

[S1] J. L. Shaneson, Wall's surgery obstruction groups for $G \times Z$, Ann. of Math. (2) 90 (1969), 296-334. MR 39 \#7614.

[S2] D. Sullivan, Geometric topology seminar notes, Mimeographed notes, Princeton Univ., 1967.

[W1] F. Waldhausen, Whitehead groups of generalized free products (Mimeographed preprint).

[W2] C. T. C. Wall, Surgery on compact manifolds, Academic Press, New York, 1970.

[M] A. S. Mišccenko, Homotopy invariants of non-simply connected manifolds, II. Simple homotopy type, Izv. Akad. Nauk SSSR Ser. Mat. 35 (1971), 655-666 = Math. USSR Izv. 5 (1971), 668-679. MR 45 \#2734.

INSTITUT DES HAUTES ÉTUDES SCIENTIFIQUES, BURS-SUR-YVETTE, FRANCE

DEPARTMENT OF MATHEMATICS, PRINCETON UNIVERSITY, PRINCETON, NEW JERSEY 08540 